\title{
Study on the Minimization Design of Microstrip Line Radiation Field
}

\author{
CHEN Jian-hua \\ School of Information Engineering \\ Henan University of Science \&Technology \\ Luoyang, China \\ e-mail:chenjh_16888@163.com
}

\author{
LI Ying \\ Jianhe Community \\ Henan petroleum exploration bureau \\ Nanyang, China
}

\begin{abstract}
Based on finite difference time domain (FDTD) method, a monoaxial anisotropic perfectly matched layer was used as absorbing boundary condition, a gauss pulse signal with a bandwidth of 0-10GHz and a peak value of $1 \mathrm{~V}$ was used as excite source, the impedance of microstrip was $50 \Omega$, excitation signal source impedance and load impedance were matched with the microstrip line characteristic impedance. Firstly, the near zone radiation field of the microstrip line was obtained via FDTD method, then, the near-zone to far-zone transformation method was used to obtain far-zone field, the far-zone field was on the surface of a sphere which had a radius of 3 meters and the centered at the origin, finally, the effects of microstrip line width and dielectric thickness of microstrip line on far-zone field were analyzed. The results can be used to minimize the radiation field produced by the microstrip line under the condition of meeting the process and technical specifications.
\end{abstract}

Keywords:-Finite-difference time-domain (FDTD); microstrip line; impedance; radiation field

\section{INTRODUCTION}

With the development of science and technology, the electronic equipments have the characteristics of high speed, wide band, high sensitivity, high density and small size. The problems caused by the electromagnetic radiation are even more prominent. Electromagnetic radiation generated by the equipment not only interferes with the function circuits inside the equipment but also the other equipments in the surrounding space, affects these equipments to work normally and even leads to major accidents in production or life, at the same time, it will cause environmental electromagnetic pollution which will affect people's physical and mental health Therefore, the developed countries have set up mandatory standards for the electromagnetic radiation of electronic equipments, if the product's radiation emission cannot meet the corresponding standards, will not be able to market sales. Therefore, electromagnetic radiation has become the current research hotspot and important research topic. The microstrip line on printed circuit board (PCB) is one of the carriers to carry signals, so it is very important to study the radiation field of microstrip line and minimize it.

In the related literatures[1-3], The radiation field of microstrip line was studied, However, there is no research on

Supported by NSFC: 61304144 how to minimize the radiation field of the microstrip line. Because the FDTD method is directly based on the Maxwell equation, the result is "complete" vector field, at the same time, the FDTD method has the characteristics of mature, reliable and accurate[4-5]. In the time domain or frequency domain, it can be used to analyze the microstrip line and other electromagnetic problems[6-8]. Therefore, in this paper, this method is used to study how to reduce the radiation field of the $50 \Omega$ microstrip line and minimize the radiation field.

In this paper, the FDTD method was used to study the microstrip circuit, a perfectly matched anisotropic absorber was used as an absorbing boundary condition (UPML) $[5,6,11,12]$, a gauss pulse was used as exciting source, the peak value and bandwidth of Gauss pulse are $1 \mathrm{~V}$ and 0 $10 \mathrm{GHz}$ respectively, the method of plane excitation network was used to excite microstrip line[9], the characteristic impedance of the microstrip line, the excitation signal source resistance and load were $50 \Omega$, That is, under the conditions of matching, based on the near zone field of the radiation field of the microstrip line obtained by the FDTD method, the near field to far field transformation method was used to study the effects of microstrips width and thickness of microstrip line dielectric on the far area radiation field, the far-zone field was on the surface of a sphere which had a radius of 3 meters and the centered at the origin, in Fig. 1. The results can be used to minimize the radiation field produced by the microstrip line and provide reference and basis for the design and optimization of microstrip line.

In the literature [5,6], the uniaxial anisotropic perfectly matched layer (UPML) absorbing boundary condition, the FDTD method with lumped elements, the excition method of microstripe line and the near field to far field transformation method were introduced in detail. In this paper, the microstrip line circuit and Gauss pulse signals were introduced emphatically, and simulation results were analyzed and summarized.

\section{MiCROSTRIP LINE STRUCTURE MODEL}

The microstrip line structure is shown in Fig. 1, Fig. 1 (a) is a top view, Fig. 1 (b) is a front view, $l_{1}$ is the length of microstrip line (PCB),$l_{2}$ is the width of PCB, $W$ is the width of the microstrip line, the microstrip line and return path planar 
are copper conductor $\left(\varepsilon_{\mathrm{r}}=1 、 \mu_{\mathrm{r}}=1 、 \sigma=5.8 \times 10^{7} \mathrm{~S} / \mathrm{m}\right)$, the thickness of the copper conductor is $t=0.1 \mathrm{~mm}$, the microstrip line is symmetrically distributed on PCB, $h, \mu_{\mathrm{r}}$ and $\varepsilon_{\mathrm{r}}$ are respectively PCB substrate thickness, relative permeability and relative dielectric constant, and $\mu_{\mathrm{r}}=1$, the dielectric loss tangent is 0.02 , port 1 and port 2 are respectively excitation signal source and load, excitation signal source impedance and load impedance are matched to the microstrip line characteristic impedance.

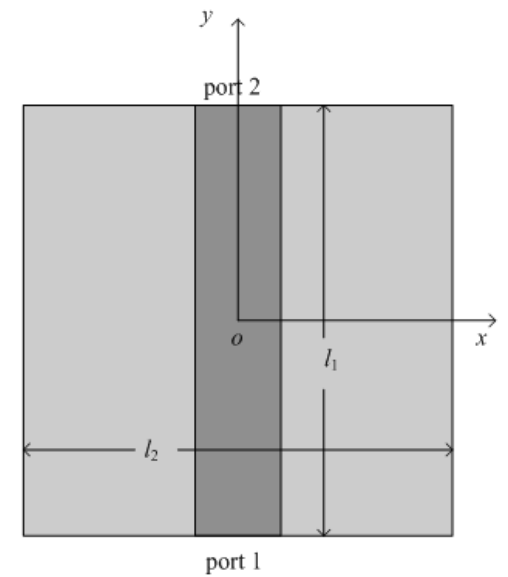

(a)

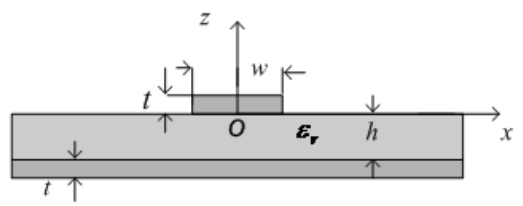

(b)

Fig. 1 PCB and microstrip line structure graph (a) Top view (b) Front view

\section{EXCITATION SIGNAL SOURCE}

When the FDTD method was used to study the radiation field of the microstrip line, the Gauss pulse was used as the excitation voltage source. The expressions of the Gauss pulse in time domain and frequency domain are respectively (1) and (2).

$$
\begin{aligned}
& V(t)=\exp \left(-\frac{4 \pi\left(t-t_{0}\right)^{2}}{\tau^{2}}\right) \\
& F(j \omega)=\frac{\tau}{2} \exp \left(-j 2 \pi f t_{0}-\frac{\pi f^{2} \tau^{2}}{4}\right)
\end{aligned}
$$

where $\tau$ is the width of the Gauss pulse, $\mathrm{t}_{0}$ is the time of the peak of the Gauss pulse, $f$ is the frequency component of the signal. In this paper, $\tau=200 \mathrm{ps}, t_{0}=150 \mathrm{ps}$. When $f=0 \mathrm{~Hz}$, the pulse spectrum amplitude has the maximum value, when $f=2 / \tau \mathrm{Hz}$, the pulse spectrum amplitude equals $4.3 \%$ of the maximum value, so $f=2 / \tau$ is usually taken as the Gauss pulse bandwidth, as a result, the signal bandwidth is $0-10 \mathrm{GHz}$ when $\tau=200$ ps .
Fig. 2 is the excitation signal source waveform when $\tau=200 \mathrm{ps}, t_{0}=150 \mathrm{ps}$.

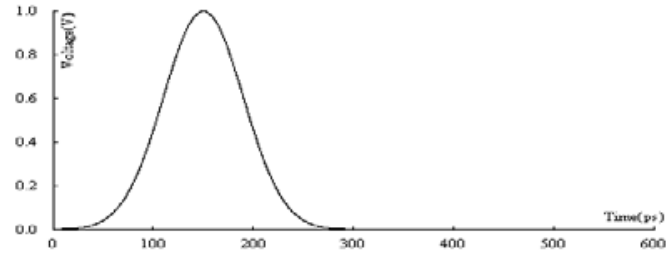

Fig. 2 excitation signal source

\section{MICROSTRIP PARAMETERS}

In order to study the effects of microstrip line structure parameters on its radiation field, we selected three sets of microstripe line with different parameters, as shown in table 1. under the condition that the characteristics impedance of microstrip line was $\mathrm{z}_{0}=50 \Omega$, the effects of the thickness of dielectric substrate of PCB and the width of the microstrip line on its radiation field were studied. The purposes were to optimize the design of microstrip line and minimize the radiation field produced by the microstrip line.

TABLE I. MICROSTRIPE LINE PARAMETERS

\begin{tabular}{cccccccc}
\hline & $W(\mathrm{~mm})$ & $H(\mathrm{~mm})$ & $\varepsilon_{\boldsymbol{r}}$ & $z_{0}(\Omega)$ & $T(\mathrm{~mm})$ & $l_{1}(\mathrm{~mm})$ & $l_{2}(\mathrm{~mm})$ \\
\hline set 1 & 0.25 & 0.3 & 9.00 & 50 & 0.1 & 50 & 13 \\
set 2 & 0.25 & 0.2 & 5.85 & 50 & 0.1 & 50 & 13 \\
set 3 & 0.50 & 0.3 & 4.48 & 50 & 0.1 & 50 & 13 \\
\hline
\end{tabular}

When FDTD method was used to analyze microstrip line, the UPML with ten layers was used as absorbing boundary condition, the plane excitation network was used to excite microstrip line, in the excitation plane, the electric field component produced by the voltage source is only in $\mathrm{Z}$ direction. According to the parameters of the microstrip line, the size of Yee's grid walsx $=0.125 \mathrm{~mm}, \Delta y=0.5 \mathrm{~mm}$, $\Delta z=0.1 \mathrm{~mm}$, the time step was $\Delta t=0.2 p s$. From the edge of the PCB to the data output boundary, there were ten layers of Yee's grid.

\section{NUMERICAL ANALYSIS RESULTS}

Because the microstrip line characteristic impedance $\mathrm{z}_{0}$ is a function, its value is decided by $w, h, \varepsilon_{r}$ and $t$. Therefore, in table 1 , under the condition of $\mathrm{z}_{0}=50 \Omega$ and $\mathrm{t}=0.1 \mathrm{~mm}$, as long as either $w$ or $h$ has a change, $\varepsilon_{r}$ will change accordingly, Therefore, when considering the difference of microstrip lines with different parameters, $\varepsilon_{r}$ is no longer considered as an independent variable.

The results showed that, for the three sets of the microstrip line with different parameters, at the excitation port 1 , the signal peak value was $500 \mathrm{mV}$, the impedance matching between the excitation source and the microstrip line was further explained. the signal peak at the load port 2 were respectively $482.4 \mathrm{mV}, 495.7 \mathrm{mV}$ and $493.5 \mathrm{mV}$, this showed that the attenuation of the signal was different with the change of the microstrip line parameters. 
As shown in Figure 1, in the $\mathrm{x}$ direction and the $\mathrm{y}$ direction, due to the PCB structure symmetry, therefore, when we studied the radiation field of the microstrip line, we chose five points at the distance of 3 meters to study the variation of the radiation field. they were $\left(r=3, \theta=0^{\circ}, \varphi=0^{\circ}\right),\left(r=3, \theta=45^{\circ}, \varphi=180^{\circ}\right)$, $\left(r=3, \theta=90^{\circ}, \varphi=180^{\circ}\right) \quad, \quad\left(r=3, \theta=45^{\circ}, \varphi=270^{\circ}\right) \quad$ and $\quad\left(r=3, \theta=90^{\circ}, \varphi=270^{\circ}\right) \quad$, respectively. According to the relationship between the spherical coordinates and Cartesian coordinates, we can get the variation of the radiation field both in the $\mathrm{x}$ direction and the $y$ direction.

For the three sets of different parameters microstrip lines, as shown in table 1 , total radiation field at foregoing five points are reported in figure 3 to 7 . By comparing the peak value of the radiation field at each point , we get the following conclusions. The radiation field has the maximum value at the point $\left(r=3, \theta=0^{\circ}, \varphi=0^{\circ}\right)$, that is, the radiation field maximum value is right above the microstrip line. In the xoz plane and yoz plane, the radiation field decrease with the increase of $\theta$, but the radiation field in the $\mathrm{y}$ direction is greater than that in the $\mathrm{x}$ direction. The other parameters of the microstrip line are kept unchanged, When the thickness of the dielectric layer is reduced from $0.3 \mathrm{~mm}$ to $0.2 \mathrm{~mm}$, or the width of the microstrip line is reduced from $0.5 \mathrm{~mm}$ to $0.25 \mathrm{~mm}$, the peak value of the radiation field decrease at the above five points. Therefore, when we design microstrip line, under the condition of meeting the performance of the circuit, The radiation field generated by the microstrip line can be minimized by minimizing the thickness of the dielectric substrate and the width of the microstrip line.

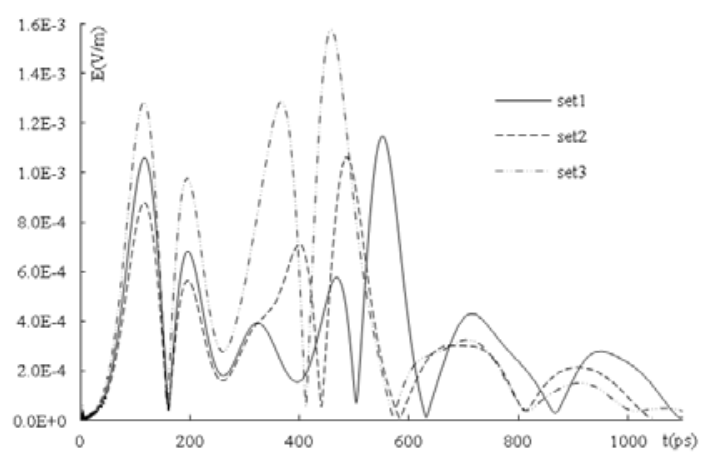

Fig. 3 Total radiation field at point $\left(r=3, \theta=0^{\circ}, \varphi=0^{\circ}\right)$

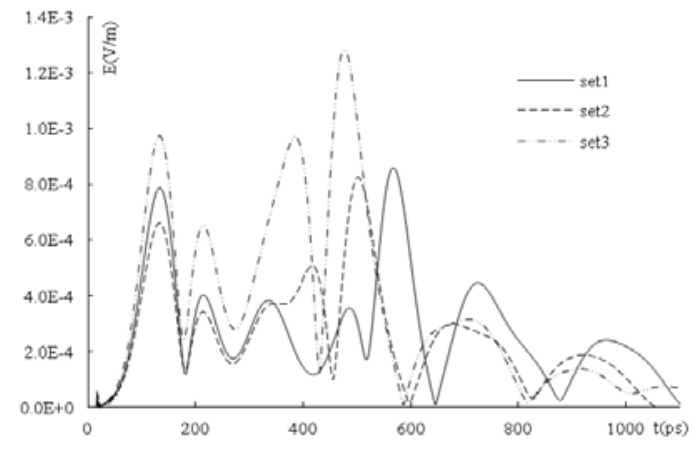

Fig. 4 Total radiation field at $\left(r=3, \theta=45^{\circ}, \varphi=180^{\circ}\right)$

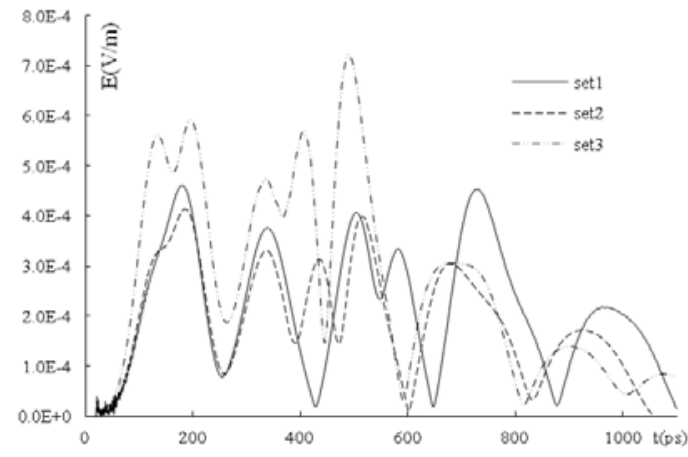

Fig. 5 Total radiation field at point $\left(r=3, \theta=90^{\circ}, \varphi=180^{\circ}\right)$

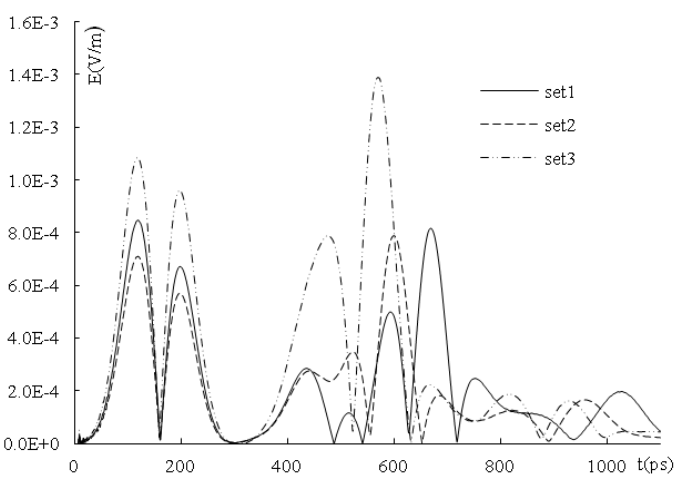

Fig. 6 Total radiation field at point $\left(r=3, \theta=45^{\circ}, \varphi=270^{\circ}\right)$

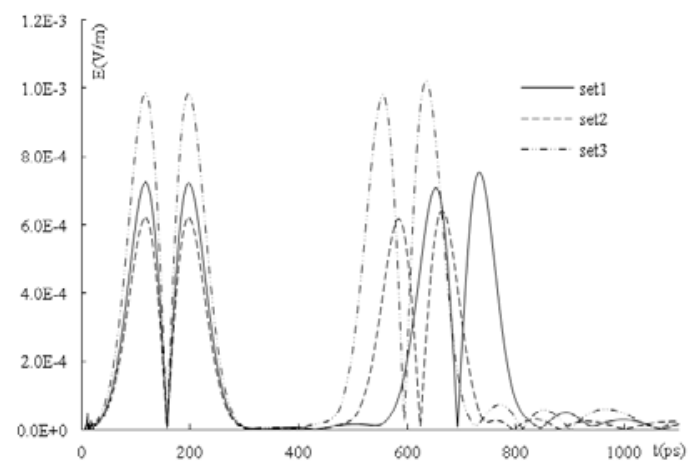

Fig. 7 Total radiation field at point $\left(r=3, \theta=90^{\circ}, \varphi=270^{\circ}\right)$

\section{CONCLUSIONS}

The microstrip line characteristic impedance was $50 \Omega$, excitation source was a Gauss pulse, the bandwidth of Gauss pulse was $0-10 \mathrm{Ghz}$, the plane excitation network was used to excite microstrip line, under the above conditions, the FDTD method was used to analyze the radiation field generated by microstrip lines with different parameters.

The results show that the radiation field maximum value was right above the microstrip line, the direction of maximum radiation field was consistent with the direction of the length of the microstrip line, In the xoz plane and yoz plane, the radiation field decreased with the increase of $\theta$, the maximum radiation field strength could be reduced by decreasing the 
thickness of the microstrip line dielectric layer and the width of the microstrip line. Therefore, in the design of microstrip line, under the premise of meeting the performance of the circuit, the radiation field generated by the microstrip line can be minimized by minimizing the thickness of the dielectric substrate of the microstrip line as well as the width of the microstrip line.

\section{References}

[1] R. Cicchetti, "Transient analysis of radiated field from electric dipoles and microstrip lines," IEEE Trans. on AP, vol. 39, n. 7, pp. 910-918, july 1991.

[2] R. Cicchetti, A. Faraone, "Transient emission from microstrip interconnects: theoretical formulation and CAD modeling,” IEEE Trans. on EMC, vol. 38, n. 3, pp. 367-375, August 1996.

[3] D.A. Hill, D.G. Camell, K.H. Cavcey,G.H. Koepke, "Radiated emissions and immunity of microstrip transmission lines: theory and reverberation chamber measurements,” IEEE Trans. on EMC, vol. 38, n. 2, pp. 165-172, May 1996.
[4] K. S. Yee, "Numerical solution of initial boundary value problems involving Maxwell's equations in isotropic media," IEEE Trans. on AP, vol. 14, n. 3, pp. 302-307, May 1966.

[5] Ge D B, Yan Y B, Finite-Difference Time-Domain Method for Electromagnetic Wav, 2rd ed., Xidian University Press, Xi’an, 2005.

[6] Chen J H, Zhou L P, Li Y, "Effect of Asymmetry of Differential Circuit on Signal Integrity and Coupling Noise," Journal of Henan University of Science and Technology, vol. 34, n. 4, pp. 45-50, April 2013.

[7] R. J. Luebbers, K. Kunz, M. Schneider, "A finite-diference time-domain near zone to far zone transformation,” IEEE Trans. on AP, vol. 39, n. 4, pp. 429-433, April 1991.

[8] T. Martin, "An improved near- to far-zone transformation for the finitediference time-domain method," IEEE Trans. on AP, vol. 46, n. 9, pp. 1263-1271, September 1998.

[9] Stephen D. Gedney, "An Anisotropic Perfectly Matched LayerAbsorbing Medium for the Truncation of FDTD Lattices,” IEEE Trans. on AP, vol. 44, n. 12, pp. 1630-1639, December 1996.

[10] Dennis M. Sullivan, “An Unsplit Step 3-D PML for Use with the FDTD Method,” IEEE microwave and guided wave letters, vol. 7, n. 7, pp. 184-186, July 1997. 